\title{
Difficult Decisions
}

\section{Whether or not to give adjuvant chemotherapy for breast} \section{cancer}

\author{
Ian E. Smith
}

Royal Marsden Hospital, Fulham Road, London SW3, UK.

\section{Introduction}

There is no longer any reasonable doubt that adjuvant chemotherapy prolongs survival for premenopausal women with early breast cancer. Indeed, in more than 100 years of research into breast cancer management it is the first form of treatment convincingly shown to have survival benefit. Yet important questions linger over its real clinical value. These include the balance of benefit against morbidity, and the possibility that the treatment effect may be largely endocrine-mediated. For many clinicians whether or not to give adjuvant chemotherapy is still a difficult decision.

\section{New evidence for survival benefit}

The rationale for adjuvant chemotherapy is a simple one. Most patients presenting with so-called early breast cancer which appears to be localized to the breast and/or regional nodes in reality already have micro-metastatic disease from which they will eventually die unless they are given effective systemic treatment as an adjunct to surgery.

The first adjuvant chemotherapy trials were started three decades ago, ${ }^{1}$ but real interest in this approach was stimulated by two important trials which were started in the 1970s. The first of these, run by the National Surgical Adjuvant Breast Project (NSABP) Group in the USA compared 2 years of oral melphalan (L-PAM) with an oral placebo in patients who were found to have axillary node involvement at surgery. The second trial, based in Milan, compared combination chemotherapy using cyclophosphamide, methotrexate and 5-fluorouracil (so-called CMF) for one year after surgery with no further treatment. Both these trials began with a bang. First results showed a signifi-

Correspondence: I.E. Smith, M.D., F.R.C.P., F.R.C.P.E. Received: 8 June 1988 cant delay in tumour recurrence for patients receiving chemotherapy ${ }^{2,3}$ and were prematurely hailed as a major advance. With time, there was an inevitable reaction to this early euphoria; since chemotherapy achieves transient tumour regression in the majority of patients with advanced disease it was really of no great surprise that a short delay in the emergence of metastatic disease would likewise be seen if used at first presentation. For most clinicians the acid test of benefit was improvement in survival itself and until this was forthcoming both trials were regarded with some scepticism.

Twelve years have now elapsed, however, and long term follow-up results have stood the test of time. In the NSABP melphalan trial a clear cut $37 \%$ reduction in mortality 10 years after treatment has emerged for treated patients under 50 years old $(60 \%$ versus $36 \%$ still alive) with a maximum benefit seen in patients with only 1-3 axillary nodes involved (64\% reduction in mortality). ${ }^{4}$ Likewise in the Milan trial, premenopausal patients treated with CMF chemotherapy have shown a significant $35 \%$ reduction in the odds of death $(59 \%$ versus $45 \%$ alive at 10 years). ${ }^{5}$ Interestingly, neither trial has shown a significant survival difference for postmenopausal patients.

Most subsequent chemotherapy trials have tended to confirm these results although the magnitude of the effect varies. For example in the UK a combined Guy's Hospital/Christie Hospital, Manchester trial has so far simply shown a small survival trend in favour of premenopausal patients treated with CMF chemotherapy which has not yet reached statistical significance $(80 \%$ versus $70 \%$ alive at 3 year median follow-up). ${ }^{6}$ Duration of follow-up may be important here. Survival benefit in the NSABP and Milan trials tended to become more pronounced with time, and it may well be that the same will happen with this and other 'less mature' trials.

(C) The Fellowship of Postgraduate Medicine, 1988 
Overall, more than 15,000 women have been entered into adjuvant chemotherapy trials with no treatment control arms throughout the world, and the most convincing evidence that survival benefit is real comes from an overview analysis of survival data from these trials. ${ }^{7}$ This confirmed an overall reduction in mortality of around $25-30 \%$ for patients under 50 treated with combination chemotherapy, usually involving CMF. In contrast the survival benefit for post-menopausal women was minimal. Because of the nature of analysis, there are so far no good data on the relationship between survival and duration of follow-up: this may come from further similar analyses in the future. Nevertheless, the statistical methods used are widely accepted as being valid and powerful and the results confirm beyond reasonable doubt that adjuvant chemotherapy does indeed achieve a significant improvement in survival for patients under 50 years old. The dilemma for the clinician lies in deciding whether this survival difference is clinically worthwhile for the patient and whether chemotherapy is the best way to improve survival.

\section{Is this survival improvement clinically worthwhile?}

A $30 \%$ reduction in mortality is not necessarily as dramatic as it sounds, and depends on the actual mortality in the control group. In the overview analysis the actual percentage difference in survival between treated and non-treated patients was estimated to be around $10 \%$ at 5 years follow-up (36\% vs $27 \%$ dead). In the Milan CMF trial the difference was $14 \%$ at 10 years, and in the NSABP trial $24 \%$ at 10 years. Whatever figure is chosen, this form of analysis implies that only a small minority of patients will benefit from treatment. This is of course a common problem for many forms of cancer treatment and does not necessarily invalidate the therapeutic approach, but it is an important factor to be taken into account when assessing clinical benefit.

Recently, it has been argued that a simple survival difference at a specific time point may not be the best way to assess overall benefit, and that what should be measured instead is the difference in median survival between treated and untreated patients. $^{8}$ This latter approach allows for many patients whose sub-clinical tumour cell burden might be sufficiently reduced by chemotherapy to prolong survival without leading to cure. Using this approach, women under 50 treated with adjuvant chemotherapy may expect to live 3-5 years longer than those not receiving such treatment. ${ }^{8}$

My own view is that each of these approaches is valid in making a value judgement about treatment benefit, providing the patient understands what is being offered. A $10 \%$ difference in survival at a certain time point may be perceived differently from 3-5 years of extra life.

\section{Morbidity of adjuvant chemotherapy}

If adjuvant chemotherapy were without morbidity then its use would be universally accepted without question or debate. Unfortunately, all forms of chemotherapy have at least some risk of side effects and it is this problem more than any other that has led clinicians to question its real clinical benefit. The problem of morbidity must therefore be looked at carefully.

The severity of chemotherapy-induced side effects depends upon many factors of which the most important are the nature and number of drugs used. Care and expertise in supervising therapy also play an important part in minimizing morbidity. Some years ago, a frequently quoted study from the Royal Marsden Hospital reported major side effects in most patients receiving adjuvant chemotherapy with almost $30 \%$ volunteering that the experience could not be gone through again. ${ }^{9}$ This study related to a 5 drug combination regimen, with treatment usually given in an over-crowded surgical out-patient clinic by over-worked junior doctors. For the last few years, a simpler 3 drug CMF regimen has been used, and treatment has been supervised in a committed medical clinic by a team of physicians and specialist nurses. Patients managed in this way have recently been assessed in the same way and by the same independent observer as in the original study. Although side effects were certainly not abolished, their incidence and severity were very markedly reduced, and most patients found that they could continue a reasonably normal life during treatment. Some comparative figures illustrate the difference between these 2 approaches: severe vomiting - first study $76 \%$, second study $4 \%$; alopecia requiring a wig $83 \%$ vs $22 \%$; severely off-colour $54 \%$ vs $4 \%$; severe effect on life-style $37 \%$ vs $9 \%$.

There are several reasons for these important improvements: these include the choice of drugs given, the use of newer techniques of anti-emetic therapy ${ }^{10,11}$ and perhaps most important of all a medical and nursing team with the time to explain, listen and provide support. Adjuvant chemotherapy, like surgery and radiotherapy, remains unpleasant for most patients and its advantages and snags undoubtedly need to be discussed with each woman being offered this treatment. However, with careful supervision it is very rarely the blight on life suggested by the media and indeed by some clin- 
icians, and a refusal even to offer treatment on such grounds is quite unjustified. Nor is the excuse that a busy surgical team does not have the time to offer chemotherapy acceptable; if a treatment for breast cancer is of real clinical benefit then time, resources and appropriate staff have to be found for its proper delivery.

\section{How does adjuvant chemotherapy work?}

Adjuvant chemotherapy was conceived on the reasonable premise that survival might be improved through a direct cytotoxic effect on micro-metastatic tumour cells. However, it is difficult on this basis to explain the recurring observation that such treatment appears to be of significant benefit only in premenopausal women. This raises the important question as to whether its effect is instead mainly endocrine-mediated through ovarian suppression.

It is well established that chemotherapy suppresses ovarian function ${ }^{12,13}$ and most patients receiving CMF chemotherapy develop amenorrhoea. ${ }^{5.6} \mathrm{~A}$ powerful argument in favour of an endocrine-mediated effect has come from a recent analysis of the Guy's-Christie Hospital CMF trial which showed that prolonged survival occurred only in patients who developed amenorrhoea with treatment. ${ }^{6}$ Likewise in this analysis, survival benefit was seen only in patients whose tumours were positive for cytoplasmic progestogen receptors (PR), and by implication therefore more likely to be endocrine sensitive. Other trials have so far provided little information on the relationship of survival to PR status, but there are more data on the relationship of amenorrhoea with survival and these tend to contradict the Guy's-Christie study. For example, amenorrhoea did not influence survival in a Danish CMF trial ${ }^{14}$ or relapse-free survival in the Milan CMF trial in which $37 \%$ of patients with amenorrhoea were relapse-free at the time of analysis compared with $32 \%$ who did not develop amenorrhoea, compared with $17 \%$ in untreated control patients. ${ }^{5}$ Data from this kind of analysis are therefore circumstantial and at present contradictory.

A second approach to this question comes from the results of trials directly assessing endocrine therapy itself as an adjuvant treatment. First results from adjuvant oophorectomy trials most of which were started many years ago failed to show a convincing survival improvement and this approach fell into disrepute. Recently, however, an overview analysis of these trials with much longer follow-up suggested results very similar to that achieved with adjuvant chemotherapy with a $29 \%$ reduction in the odds of death. ${ }^{7}$ This analysis has to be viewed with caution since the number of patients in oophorectomy trials are very considerably less than for chemotherapy.

In a similar context, two UK multi-centre trials of adjuvant tamoxifen have shown small but significant survival benefits for premenopausal patients with this simple treatment, with follow-up of up to 6 and 8 years. ${ }^{16}$

The extent to which the effects of endocrine therapy are indeed endocrine-mediated remains an open question, although current evidence suggests that it may well be at least a factor. Age may be an important determinant here since older premenopausal patients have around a $90 \%$ incidence of drug-induced amenorrhoea compared with a $<20 \%$ risk for patients less than 35 years old. Yet there is nothing to suggest that these younger patients do significantly less well with this treatment. There is a real need for randomized trials comparing adjuvant chemotherapy with oophorectomy to give a straight answer to this question, and these are now underway. However, there is an important caveat here: it is by no means certain in my mind that oophorectomy with its problems of irreversibility, postmenopausal symptoms, and risk of osteoporosis would be preferable to the short term side effects of chemotherapy, even if it proved to be as effective. For this reason further data are also required on the comparative merits of medical endocrine treatments including tamoxifen and the new LHRH analogues.

\section{Other problems with adjuvant chemotherapy}

Treatment duration For many patients morbidity, however trivial during the first few courses, becomes an increasing problem with long term treatment. For patients and indeed for doctors therefore the shorter the duration of chemotherapy the better. Early trials employed one or two years of treatment, but it is now becoming clear that much shorter durations of therapy are as effective. ${ }^{5,17,18}$ Conventional practice at present is to use a maximum of 6 courses of CMF chemotherapy over a 6 month period, and current trials are investigating whether even shorter courses might be as effective.

Node-negative patients Seventy five per cent of node-negative patients can expect to be alive 10 years after their diagnosis without any adjuvant treatment. There is therefore an understandable reluctance to use adjuvant chemotherapy as a general policy here. However, attempts have been made to identify the remaining $25 \%$ of patients who do badly, for example by histological grade or the absence of cytoplasmic oestrogen receptors (ER) within tumour cells, in the hope that such 
patients might benefit from adjuvant chemotherapy. Preliminary results from one trial suggest that CMF chemotherapy may indeed improve survival for node-negative patients with ER negative tumours. ${ }^{19}$

Post-menopausal patients Overall, adjuvant chemotherapy is at present contraindicated in this large group of patients, because of the absence of established benefit. Whether or not sub-groups of such patients with bad histological grade or ER negative tumours might indeed have survival improved with such treatment remains a question which may be answered from more detailed analysis of trials which are currently maturing.

\section{Conclusions}

In the uncertain world of breast cancer research,

\section{References}

1. Fisher, B., Ravdin, R.G., Ausman, R.K., Slack, N.H., Moore, G.E. \& Noer, R.J. Surgical adjuvant chemotherapy in cancer of the breast: results of a decade of cooperative investigation. Ann Surg 1968, 168: 337-356.

2. Fisher, B., Carbone, P., Economou, S.G. et al. 1-Phenylalanine mustard (L-PAM) in the management of primary breast cancer; a report of early findings. $N$ Engl J Med 1975, 292: 117-122.

3. Bonadonna, G., Brusamolino, E., Valagussa, P. et al. Combination chemotherapy as an adjuvant treatment in operable breast cancer. $N$ Engl J Med 1976, 294: 405-410.

4. Fisher, B., Redmond, C., Fisher, E.R. \& Wolmark, N. Systemic adjuvant therapy in treatment of primary operable breast cancer: National Surgical Adjuvant Breast and Bowel Project experience. NCI Monographs 1, Proceedings of the NIH Consensus Development Conference of Adjuvant Chemotherapy and Endocrine Therapy for Breast Cancer. National Cancer Institute, pp. 35-43. 1986.

5. Bonadonna, G., Valagussa, B.S., Rossi, A. et al. Tenyear experience with CMF-based adjuvant chemotherapy in resectable breast cancer. Breast Cancer Res Treat 1985, 5: 95-115.

6. Padmanabhan, N., Howell, A. \& Rubens, R.D. Mechanism of action of adjuvant chemotherapy in early breast cancer. Lancet 1986, ii: 411-414.

7. Breast Cancer Trials Co-ordinating Sub-Committee, UICC, WHO. Breast cancer trials review meeting. Washington, Sept. 1985 (unpublished).

8. Henderson, I.C. Adjuvant chemotherapy for breast cancer. $N$ Engl J Med 1988, 318: 443-444.

9. Palmer, B.V., Walsh, G.A., McKinna, J.A. \& Greening, W.P. Adjuvant chemotherapy for breast cancer: side effects and quality of life. $\mathrm{Br} \operatorname{Med} J$ 1980, 281: 1594-1597.

10. Fiore, J.J. \& Gralla, R.J. Pharmacological treatment of chemotherapy-induced nausea and vomiting. Cancer Investigation 1984, 2: 351-361.

11. Markman, M., Sheidler, V., Ettinger, D.S., Quaskey, S.A. \& Mellits, E.D. Antiemetic efficacy of dexamethasone. Randomised, double-blind, crossover study with prochlorperazine in patients receiving cancer chemotherapy. $N$ Engl J Med 1984, 311: 549-552. there is very strong evidence that adjuvant chemotherapy significantly prolongs survival for premenopausal patients with node-positive disease. Whether or not this is of real clinical benefit when balanced against toxicity is not simply a medical question but requires a value judgement from both clinician and patient; in this context, correct supervision of treatment can go a long way towards minimising side effects. Future developments with less toxic drugs or shorter duration treatment may make chemotherapy easier; conversely, simple forms of endocrine therapy may eventually be shown to be almost as effective. As trials continue, open mindedness in assessing new information as it emerges remains the key to coping with these difficult decisions.

12. Samaan, N.A., DeAsis, D.N. Jr., Buzdar, A.U. \& Blumenschein, G.R. Pituitary-ovarian function in breast cancer patients on adjuvant chemoimmunotherapy. Cancer 1978, 41: 2084-2087.

13. Rose, D.P. \& David, T.E. Effects of adjuvant chemohormonal therapy on the ovarian and adrenal function of breast cancer patients. Cancer Res 1980, 40: 4043-4047.

14. Brincker, H., Mourisden, H.T., Rank, F. et al. Evidence of a castration-mediated effect of adjuvant cyclophosphamide monotherapy v. CMF in premenopausal stage II breast cancer. J Clin Oncol 1987, 5: 1771-1778.

15. Breast Cancer Trials Committee, Scottish Cancer Trials Office. Adjuvant tamoxifen in the management of operable breast cancer: the Scottish trial. Lancet 1987, ii: 171-175.

16. Baum, M., Brinkley, D.M., Dosset, J.A. et al. Controlled trial of tamoxifen as a single adjuvant agent in the management of early breast cancer. $\mathrm{Br} J$ Cancer 1988, 57, 608-611.

17. Jungi, W.F., Alberto, P., Brunner, K.W. et al. Shortor long-term chemotherapy for node-positive breast cancer: LMF 6 versus 18 cycles. SAKK study 27/76. Recent Results Cancer Res 1984, 96: 175-177.

18. Henderson, I.C., Gelman, R.S., Harris, J.R. \& Canellos, G.P. Duration of therapy in adjuvant chemotherapy trials. NCI Monographs 1, Proceedings of the NIH Consensus Development Conference on Adjuvant Chemotherapy and Endocrine Therapy for Breast Cancer. National Cancer Institute, pp 95-98, 1986.

19. Bonadonna, G., Valagussa, P., Tancini, G. et al. Current status of Milan adjuvant chemotherapy trials for node-positive and node-negative breast cancer. NCI Monographs 1, Proceedings of the NIH Consensus Development Conference on Adjuvant Chemotherapy and Endocrine Therapy for Breast Cancer. National Cancer Institute, pp 45-49, 1986. 